\title{
Tabellen- und Abbildungsverzeichnis
}

\section{Abbildungen}

Abb. 1.1: Evidenztypen adaptiert nach Willett (1988) - 27

Abb. 1.2: Ergebnisse der Pilotstudie - 45

Abb. 1.3: Ergebnisse des Experiments $1-\mathbf{5 1}$

Abb. 1.4: Experiment 2-Ausdruck und Konstruktion - 60

Abb. 1.5: Experiment 2 - Evidenztyp und Ausdruck bei höherer Verlässlichkeit - 61

Abb. 1.6: Experiment 2 - Evidenztyp und Ausdruck bei geringerer Verlässlichkeit -61

Abb. 1.7: Experiment 3 - Evidenztyp und Konstruktion bei offensichtlich -68

Abb. 1.8: Experiment 3 - Evidenztyp und Konstruktion bei anscheinend / Anschein 68

Abb. 1.9: Experiment 3 - Evidenztyp und Ausdruck - 69

Abb. 5.1: Relative Häufigkeit von offenbar im DTA - 305

Abb. 5.2: Relative Häufigkeit von scheinbar im DTA - 306

Abb. 5.3: Relative Häufigkeit von anscheinend im DTA - 309

Abb. 5.4: Relative Häufigkeit von offensichtlich im DTA -309

\section{Tabellen}

Tab. 1: Leithypothesen zu evidentiellen Satzadverbien - 3

Tab. 1.1: Absolute Häufigkeiten im Vergleich — 10

Tab. 1.2: Hypothesen des ersten Kapitels - 12

Tab. 1.3: Kombinatorik von offen mit sichtlich und sichtbar -22

Tab. 1.4: Wortstellung sichtlich versus offensichtlich -22

Tab. 1.5: Evidentiale und Commitment -43

Tab. 1.6: Experiment 1 - Bedingungen - 49

Tab. 1.7: Experiment 2-Zielsatzbedingungen - $\mathbf{5 5}$

Tab. 1.8: Experiment 3 - Zielsatzbedingungen - 64

Tab. 2.1: Hypothesen des zweiten Kapitels — 91

Tab. 2.2: Vorhersagen der SAdv-Lizenzierungshypothese in zwei Varianten - 92

Tab. 2.3: Vorhersagen der Hypothesen und Ergebnisse der Untersuchung $-\mathbf{1 6 0}$ 
Tab. 3.1: Fragestellungen und Hypothese des dritten Kapitels -164

Tab. 3.2: Skopus, Projektion und Fragen bei SAdv, komplexen Adverbialen und Matrixsätzen im Vergleich - 203

Tab. 3.3: Projektion bei SAdv und bei Evidentialen - $\mathbf{2 1 3}$

Tab. 3.4: Sprachübergreifende Testergebnisse für Evidentiale - 213

Tab. 4.1: Vorhersagen der NAl-Ansätze - 226

Tab. 4.2: Hypothesen des vierten Kapitels - 227

Tab. 4.3: Taxonomie von Ansatztypen nach Problemlösung — 284

Tab. 4.4: Eine NAI-Taxonomie - 288

Tab. 5.1: Hypothesen des fünften Kapitels - 291

Tab. 5.2: Lehmanns Parameter 296

Tab. 5.3: Evidentielle SAdv nach Lehmanns Parametern — 299

Tab. 5.4: Schwache und starke Subjektivität bei Traugott - 301

Tab.5.5: Generative Ökonomieprinzipien bei der Entwicklung evidentieller SAdv -320

Tab. 5.6: Entwicklung der SAdv nach Ebenen - 330 